\title{
Angiographic co-registration of instantaneous wave-free ratio and intravascular ultrasound improves functional assessment of borderline lesions in the coronary artery
}

\author{
Jerzy Sacha, Przemysław Lipski, Piotr Feusette \\ Department of Cardiology, University Hospital, University of Opole, Poland
}

Adv Interv Cardiol 2018; 14, 1 (51): 107-108

DOI: https://doi.org/10.5114/aic.2018.74366

Instantaneous wave-free ratio (iFR) is a newly validated technique for evaluation of the functional severity of coronary artery disease [1, 2]. Particularly, iFR pullback assessment may map the ischemic contribution of each lesion within the same artery and provide guidance for percutaneous coronary interventions ( $\mathrm{PCI}$ ) [3]. However, standard manual or motorized pullback of a pressure guidewire with continuous iFR measurement can be associated with some inaccuracies when matching the iFR values with corresponding angiographic coronary lesions. Recently, the real-time co-registration of iFR measurements with their anatomical location has been introduced, significantly simplifying the overall examination and improving its accuracy. Moreover, the same concept of co-registration has also been employed for intravascular ultrasound (IVUS) and coronary angiography [4].

Herein, we present our first experience with the SyncVision Volcano system, which provides angiographic co-registration of both iFR and IVUS.

Our patient was an 83-year-old man who underwent primary $\mathrm{PCl}$ of the right coronary artery and was scheduled for the physiological assessment of a borderline lesion within the left anterior descending artery (LAD) (Figure $1 \mathrm{~A}$ ). The measurement of fractional flow reserve (FFR) during adenosine infusion into the right femoral vein $(140 \mu \mathrm{g} / \mathrm{kg} / \mathrm{min})$ yielded the value of 0.73 . Distal iFR was 0.83; however, the pullback iFR assessment revealed steady growth of its value throughout $L A D$ and one bigger increase $(\Delta 0.07)$ in the proximal part of the artery (Figure $1 \mathrm{~B}$ - see the diagram). This angiographic image and the corresponding iFR data indicated that there was a diffuse flow restriction within the distal and medial LAD and a tighter obstruction within the proximal LAD. Intravascular ultrasound with angiographic co-registration showed insignificant plaques in the distal and medial portion and a significant lesion in the proximal part of the LAD with minimal lumen area of $3.9 \mathrm{~mm}^{2}$ (Figure $1 \mathrm{C}$ ). Balloon pre-dilatation was performed with an NC Trek balloon (Abbott) 3.5/20 mm and then a drug-eluting stent Orsiro (Biotornik) 3.5/40 mm was implanted within the proximal $L A D$, and finally post-dilatation with an NC Trek balloon (Abbott) 4.0/20 mm was done. Repeated IVUS examination with angiographic co-registration revealed a good stent expansion and no edge dissection (Figure $1 \mathrm{D}$ ). The final functional measurements revealed residual flow restriction with iFR equal to 0.9 and FFR equal to 0.86 , probably due to diffuse insignificant stenosis in the medial and distal part of the LAD. Of note, the iFR estimation before PCI anticipated that after removal of the proximal stenosis (marked with a white line on the LAD in Figure $1 \mathrm{~B}$ ), the final distal iFR value would increase to 0.9 , and this indeed happened. On the next day, the patient was discharged home in a good condition. This case shows that the real-time co-registration of different diagnostic modalities is a useful approach in coronary interventions. Particularly, the combination of imaging and functional techniques significantly increases the diagnostic and therapeutic accuracy.

\section{Conflict of interest}

The authors declare no conflict of interest. 

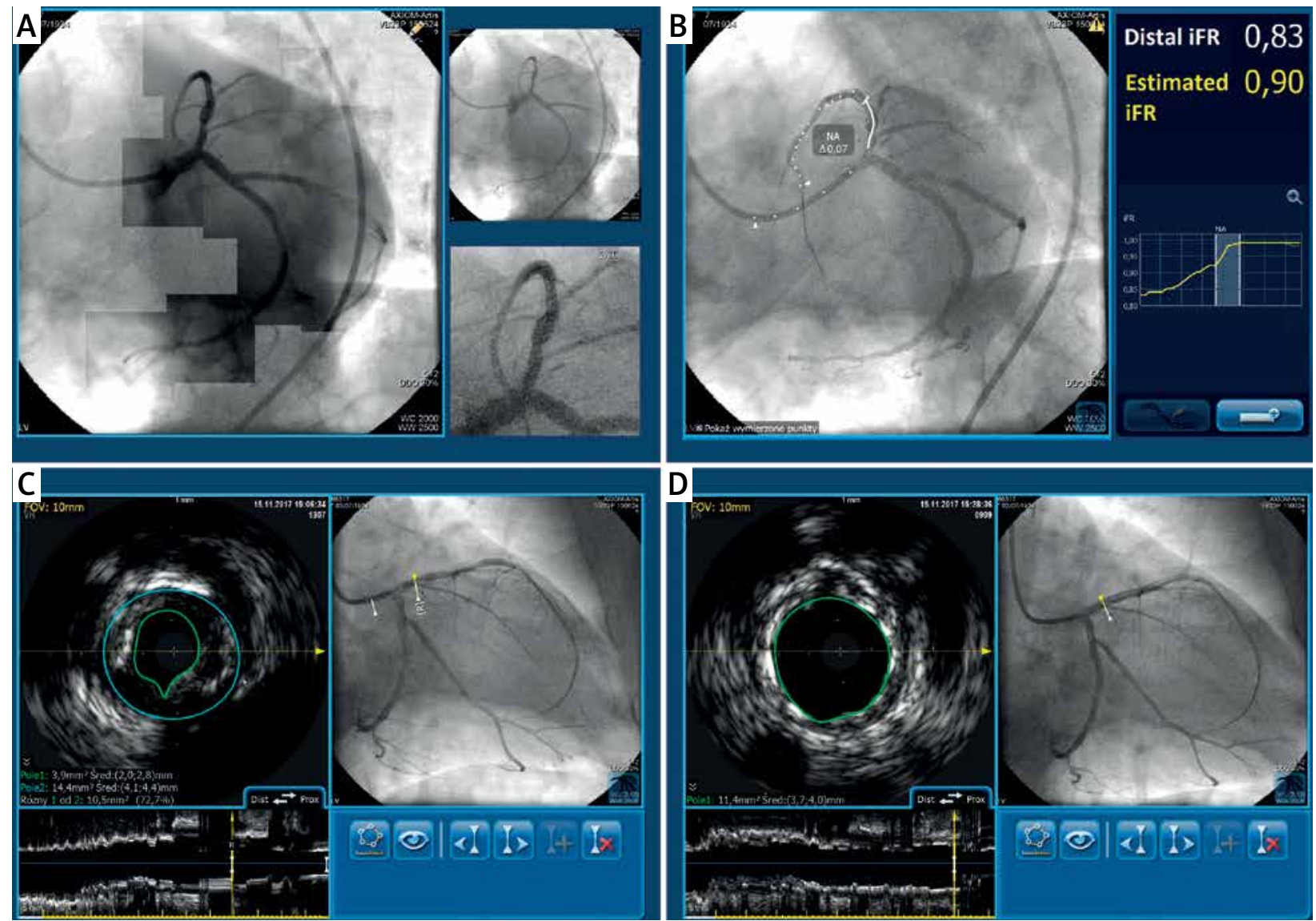

Figure 1. A - Left coronary artery angiography (spider view) shows a borderline proximal lesion in the LAD

(SyncVision system improved image quality on the largest panel). B - Angiographic co-registration of iFR: white points along LAD correspond to the consecutive iFR values shown on the diagram on the right; white line along proximal part of LAD corresponds to the steeper increase in iFR marked on the diagram $(\Delta 0.07)$; in the right upper corner the following values are given: distal iFR equals 0.83 and estimated iFR equals 0.9 , which is an anticipated iFR value after eventual removal of the lesion marked with a white line on the angiogram. C - Angiographic co-registration of IVUS presents significant stenosis in the proximal part of the LAD with minimal lumen area of $3.9 \mathrm{~mm}^{2}$. D - Angiographic co-registration of IVUS shows the same site in the LAD as in panel C but after $\mathrm{PCl}$

\section{References}

1. Davies JE, Sen S, Dehbi HM, et al. Use of the instantaneous wave-free ratio or fractional flow reserve in $\mathrm{PCl}$. N Engl J Med 2017; 376: 1824-34.

2. Götberg M, Christiansen EH, Gudmundsdottir IJ, et al. Instantaneous wave-free ratio versus fractional flow reserve to guide PCI. N Engl J Med 2017; 376: 1813-23.

3. Nijjer SS, Sen S, Petraco R, et al. The Instantaneous wave-Free Ratio (iFR) pullback: a novel innovation using baseline physiology to optimise coronary angioplasty in tandem lesions. Cardiovasc Revasc Med 2015; 16: 167-71.

4. Frimerman A, Abergel E, Blondheim DS, et al. Novel method for real time co-registration of IVUS and coronary angiography. J Interv Cardiol 2016; 29: 225-31. 